\title{
USP8 is a Novel Therapeutic Target in Melanoma Through Regulating Receptor Tyrosine Kinase Levels
}

\author{
Baoxue Duan',* \\ Changying Wang ${ }^{2, *}$ \\ Zeng Liu (iD ${ }^{3}$ \\ Xiaoyu Yang ${ }^{2}$
}

'Department of Dermatology, Xiangyang No.I People's Hospital, Hubei University of Medicine, Xiangyang, Hubei, People's Republic of China; ${ }^{2}$ Department of Oncology, Xiangyang No.I People's Hospital, Hubei University of Medicine, Xiangyang, Hubei, People's Republic of China; ${ }^{3}$ Department of Oncology, Xiangyang Central Hospital, Affiliated Hospital of Hubei University of Arts and Science, Xiangyang, Hubei, People's Republic of China

*These authors contributed equally to this work
Correspondence: Zeng Liu; Xiaoyu Yang Email liuzeng56@I63.com;

12359521@qq.com
Introduction: The hyperactivation of receptor tyrosine kinase (RTK)-mediated pathways plays an important role in melanoma progression and resistance to therapy. The ubiquitinspecific protease 8 (USP8) is a deubiquitinating enzyme and its inhibition induces degradation of RTKs. This work explored the expression and role of USP8 in melanoma.

Methods: ELISA and qPCR were performed to assess USP8 expression in melanoma tissues and cells, as well as their normal counterparts. Cellular proliferation, migration and apoptosis assays were performed to determine USP8 functions in three melanoma cell lines. Western blot was performed to analyze RTK signaling in melanoma cells after USP8 inhibition.

Results: mRNA and protein level of USP8 were higher in melanoma cells than normal melanocytes. Higher USP8 expression was also found in tumors in the majority of melanoma patients. USP8 expression was not associated with clinicopathological features, such as age, disease stage, histology, ulceration and BRAF status. Functional analysis demonstrated that USP8 overexpression promoted melanoma cell activities and alleviated the inhibitory effects of therapeutic drugs. In contrast, USP8 knockdown suppressed melanoma cell growth, survival and migration, and augmented the inhibitory effects of therapeutic drugs. Mechanism studies revealed that USP8 inhibition remarkably reduced the expression level of multiple oncogenic RTKs, including c-Met, Kit, EGFR and GPCR. Consistently, RTKmediated downstream pathways were disrupted in USP8-depleted cells, leading to the increased level of pro-apoptotic proteins and decreased level of anti-apoptotic proteins.

Conclusion: Inhibition of USP8 activity is a novel sensitizing strategy to overcome therapy resistance in melanoma.

Keywords: melanoma, receptor tyrosine kinase, USP8, therapy resistance

\section{Introduction}

Melanoma is the most lethal form of skin cancer and its incidence has been gradually increasing over the past few decades. ${ }^{1}$ Melanoma is insensitive to conventional chemotherapy and the prognosis is very poor, particularly in advanced stages. Mutations in proto-oncogene BRAF are identified in 50\% of metastatic melanoma cases. Vemurafenib, B-Raf inhibitor, has been approved for the clinical treatment of BRAF-mutant metastatic melanoma. Although the introduction of vemurafenib has significantly improved the clinical outcome, patients still develop resistance, either primary or acquired to this therapy. ${ }^{2}$ There is a need to identify novel therapeutic strategies with less-toxic, targeted treatments to overcome resistance for metastatic melanoma. 
Ubiquitin-specific proteases (USPs) are the largest deubiquitinating enzymes family and are critically involved in several human pathologies including tumorigenesis and cancer progression. ${ }^{3,4}$ USPs are emerging as promising targets for drug design due to their highly specific activity in controlling the ubiquitin-proteasome system. ${ }^{5}$ Ubiquitin-specific protease 8 (USP8), one of USP family member, has been recently revealed to play important roles in cancer progression, metastasis and drug resistance. ${ }^{6-9}$ USP8 interacts with Cdc25, Nrdp1, ErbB2 and epithelial growth factor receptor (EGFR), which are all clinically relevant cancer targets. USP8 knockout mice display significant growth retardation and marked downregulation of several receptor tyrosine kinases (RTKs), including EGFR, c-Met and ErbB3. ${ }^{10}$ Importantly, USP8 inhibition suppresses proliferation and invasion, and induces death in many cancers via reducing RTKs level. ${ }^{8,11,12}$ Melanoma has significantly elevated RTKs than normal melanocytes and their downstream signaling play key roles in melanoma development. ${ }^{13}$ However, little is known on the role of USP8 in melanoma. We hypothesized that USP8 might be a promising therapeutic target in melanoma.

In this work, we compared melanoma cells and tissues with their normal counterparts on USP8 expression. We then identified the role of USP8 in melanoma cells using gain-of-function and loss-of-function approaches. We finally investigated whether USP8 inhibition affected RTKs in melanoma cells.

\section{Materials and Methods}

\section{Primary Tissue Samples, Cell Lines and Drugs}

Frozen normal skin and melanoma tissue samples were obtained from Department of tissue repository in Xiangyang No. 1 People's Hospital. All participants provided written informed consent prior to commencement of the study. This was taken during participants' visit to Xiangyang Central Hospital and all recruitment protocols were approved by the institutional review board. Approval of tissue access was certified by the committee members of Xiangyang Central Hospital review board. Human melanoma cell lines UACC-257, UACC-62 and SK-MEL-2 were obtained from the American Type Culture Collection. Human melanoma cell lines SK-MEL-5 and SK-MEL28 were obtained from the Cell Bank of Type Culture Collection of Chinese Academy of Sciences. All cell lines were authenticated using short tandem repeat profiling
(Precision Genomics Biotechnology, Inc.). Cells used for experiments were in exponential growth phase and were examined for mycoplasma using a MycoAlert Mycoplasma Detection kit (Lonza, Inc.). Primary normal human epidermal melanocyte (NHEM\#1 and \#2) were obtained from PromoCell Inc. and Merck \& Co., Inc. Melanoma cells were cultured in MEM medium containing fetal bovine serum (FBS) to a final concentration of $10 \%$ and penicillin/streptomycin to a final concentration of $100 \mathrm{U} / \mathrm{mL}$ (Thermo Fisher Scientific, Inc.) in $95 \%$ air, $5 \% \mathrm{CO}_{2}$ and $37^{\circ} \mathrm{C}$ atmosphere. Veratridine (R\&D Systems, Inc) and vemurafenib (LC Laboratories, Inc.) were reconstituted in dimethyl sulfoxide (DMSO) and stored in $-20^{\circ} \mathrm{C}$ in aliquot.

\section{Western Blot and Enzyme-Linked Immunosorbent Assay (ELISA)}

Total protein extraction was performed using RIPA lysis buffer containing $1 \%$ protease inhibitor cocktail and phosphatase inhibitors (Thermo Fisher Scientific, Inc.). Protein concentration was measured using BCA protein assay kit (Pierce, Inc.). Twenty micrograms of protein were loaded to denaturing sodium dodecyl sulfate polyacrylamide gel and resolved via electrophoresis. Western blot was conducted according to standard protocol using primary and conjugated secondary antibodies. All antibodies were purchased from Cell Signaling, except secondary antibodies and anti- $\beta$-actin antibody (Santa Cruz Biotechnology, Inc.). Cellular and tissue USP8 levels were assessed using lysates from cells or tissues and were measured using USP8 ELISA kit (Abbexa Ltd) as per manufacturer's instructions.

\section{Measurement of Growth, Apoptosis and Migration}

Drug treatment was performed at 96-well plate and 6-well plate for growth and apoptosis. After 3 days incubation, BrdU was added to the well and cell proliferation activity was measured using BrdU Proliferation Assay (Abcam, Inc.). For apoptosis, cells were detached and resuspended in PBS, followed by Annexin V-FITC and 7-AAD (R\&D Systems, Inc.) staining. Stained cells were analyzed on MACSQuant analyzer. Migration was performed using CytoSelect 24-well cell migration assay with slight modification. Briefly, $300 \mu \mathrm{L}$ of $10^{5}$ cells $/ \mathrm{mL}$ in serum-free media with or without drug were added to the inside of each insert. Five hundred microliters of media containing $10 \%$ FBS were added to the lower well of the migration plate. After 6-hours incubation, non-migratory 
cells on the upper membrane of the insert were removed with a cotton swab. Migratory cells on the lower membrane of the insert were fixed with $10 \%$ formalin and stained with Giemsa.

\section{RT-PCR}

Total RNA was isolated from cells or tissues by using RNeasy Mini kit (Qiagen, Inc.) and converted to cDNA using iScript cDNA synthesis (Bio-Rad Laboratories, Ltd.). USP8 mRNA expression levels were quantified by using CFX96 RT-PCR machine with SYBR Green Supermix. The primer sequences of USP8 and $\beta$-actin are; F 5'-TCTCAGTGTTCCTGAAGAAGC-3' and R 5'CTAAACCAGTCAAGAAGTACC-3'; F 5'-CAACCGcG AGAAGATGA-3' and R 5'-GTCCATCACGAT GCCAGT-3'

\section{USP8 Knockdown and Overexpression}

USP8 depletion and overexpression were achieved via transfecting cells with siRNA or plasmid using Dharmafect Transfection Reagent. Transfection was performed using the same method as described in our previous study. ${ }^{14}$ USP8 siRNA (siUSP8\#1 and siUSP8\#2) and control siRNA (siCtrl) were purchased from GenePharma Inc. Cells were harvested for cellular assays at 48-h post-transfection. USP8-overexpressing plasmid was constructed via cloning USP8 cDNA to pCMV-HA (Addgene Inc.). USP8 knockdown or overexpression was confirmed by immunoblotting or ELISA.

\section{Statistical Analyses}

An unpaired Student $t$-test was used to compare data of two categorical variables. A one-way analysis of variance was performed to address if mean differences between multiple groups were significant. These compares different groups to address statistical significance. P-value $<0.05$ was defined as statistically significant.

\section{Results}

\section{USP8 Expression is Higher in Melanoma Than Normal Counterparts}

To investigate the USP8 expression in melanoma, we performed quantitative PCR, Western blotting and ELISA assay to measure its mRNA and protein level. Melanoma cell lines have been shown to recapitulate the mutational and transcriptional profiles of patient tumors. ${ }^{15}$ Five melanoma cell lines we selected for USP8 expression analysis have high correlation coefficients of patient tumors and therefore represent good models of melanoma. In addition, these cell lines cover board range of cellular origin and genetic profiling as they are derived from primary or metastasis melanoma tumors and harbor various mutations of essential oncogenic, such as B-Raf and N-Ras. As shown in Figure 1A, USP8 mRNA levels varied with the highest in SK-MEL-28 and the lowest in UACC-257 among five tested melanoma cell lines. USP8 mRNA levels were similar among different normal epidermal melanocyte samples (NHEM\#1 and NHEM\#2). Of note, 4 out of 5 melanoma cell lines displayed significant higher mRNA level on USP8 than normal melanocytes. The similar expression pattern is not limited to mRNA; USP8 protein level was significantly higher in melanoma cells $(\mathrm{P}<0.05$; Figure 1B and Supplementary Figure 1A and $\underline{B})$, suggesting that melanoma cells have higher USP8 expression level compared to normal melanocytes.

To investigate whether the expression feature observed in in vitro cells is reproducible in in vivo patients, we measured the USP8 mRNA and protein levels in a total of 22 match normal and tumor tissues obtained from melanoma patients. Each paired tumor and normal is from the same patient. Similarly, scatter plot showed that USP8 mRNA and protein levels varied among melanoma tissues and normal counterparts (Figure 2A and $\mathrm{B}$ ). The ratio of USP8 mRNA and
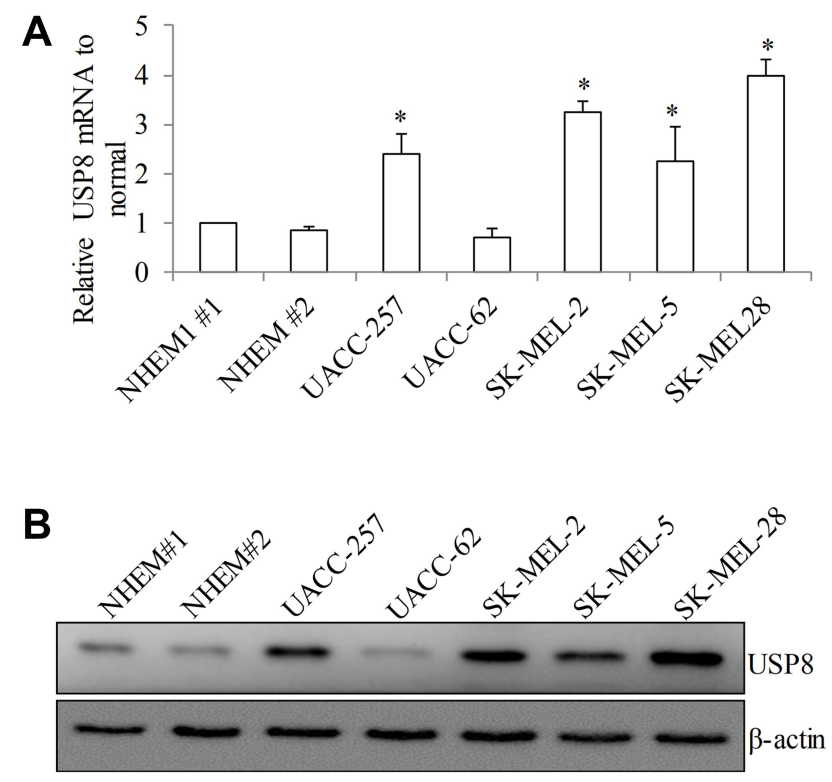

Figure I USP8 expression is significantly increased in melanoma cell lines. USP8 transcript level as assessed by RT-PCR (A) and protein level as assessed by Western blotting (B) in normal epidermal melanocytes (NHEM\#I and NHEM \#2) and a panel of melanoma cell lines (UACC-257, UACC-62, SK-MEL-2, SK-MEL-5 and SK-MEL-28). Equal amount of proteins from each sample were used in ELISA assay. Results were presented as fold change relative to NHEM\#I. $* \mathrm{P}<0.05$, compared to NHEM\#I. 
protein levels in tumor and paired normal from each melanoma patient was calculated by tumor/normal and shown in Figure $2 \mathrm{C}$ and E. The results demonstrated 13 melanoma patients with ratio at $>2 ; 3$ melanoma patients with ratio at $<1$ and 6 melanoma patients with ratio at $1 \sim 2$. The average of USP 8 mRNA and protein in melanoma was at least 2-fold higher than normal $(\mathrm{P}<0.05$; Figure $2 \mathrm{D}$ and $\mathrm{F})$. We further performed correlation analysis of USP8 expression with clinicopathological characteristics, such as age, disease stage, BRAF status, ulceration and histological classification (Supplementary Table 1). We found that UPS8 upregulation was regardless of any clinicopathological characteristics.

A

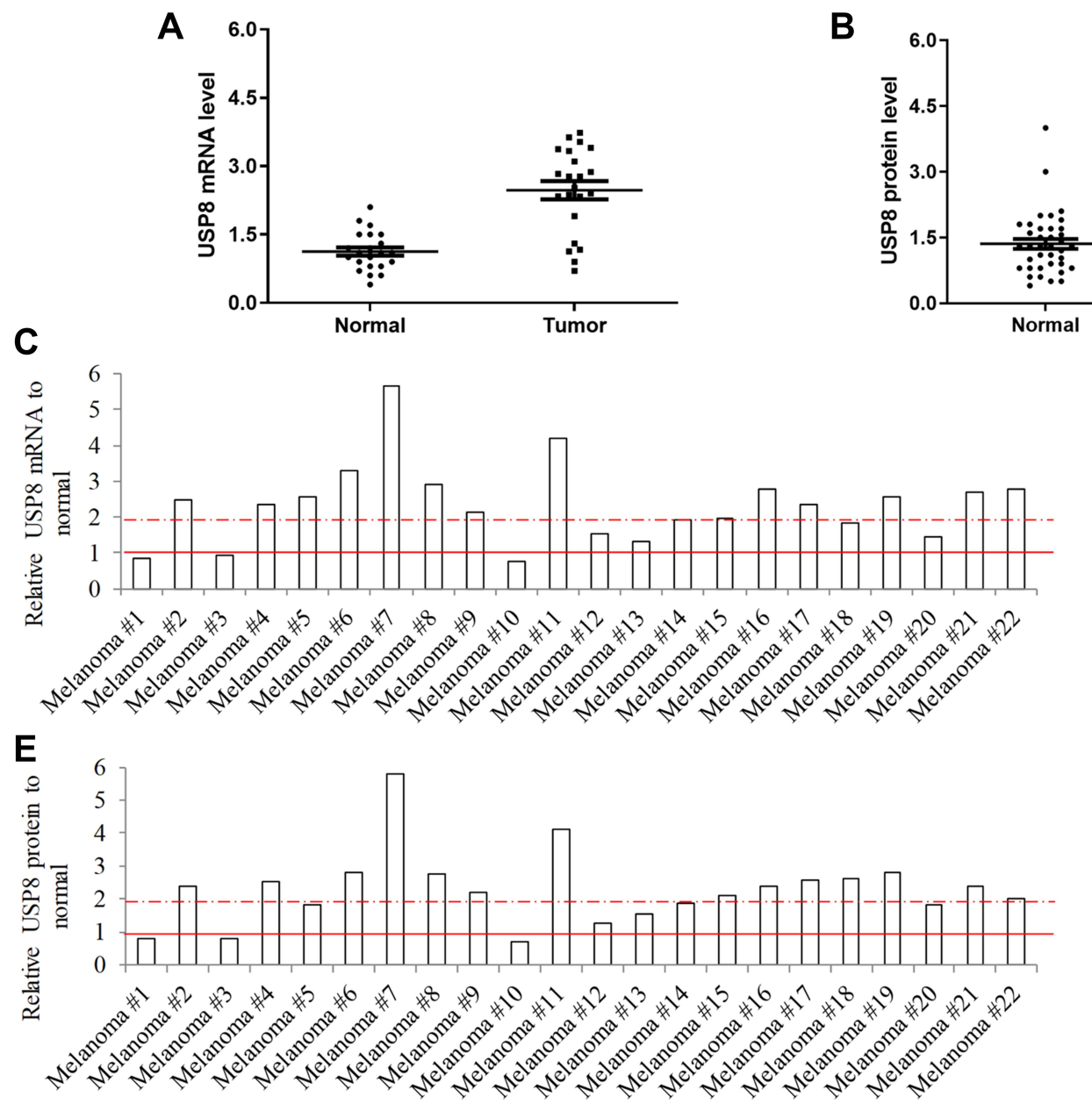

C

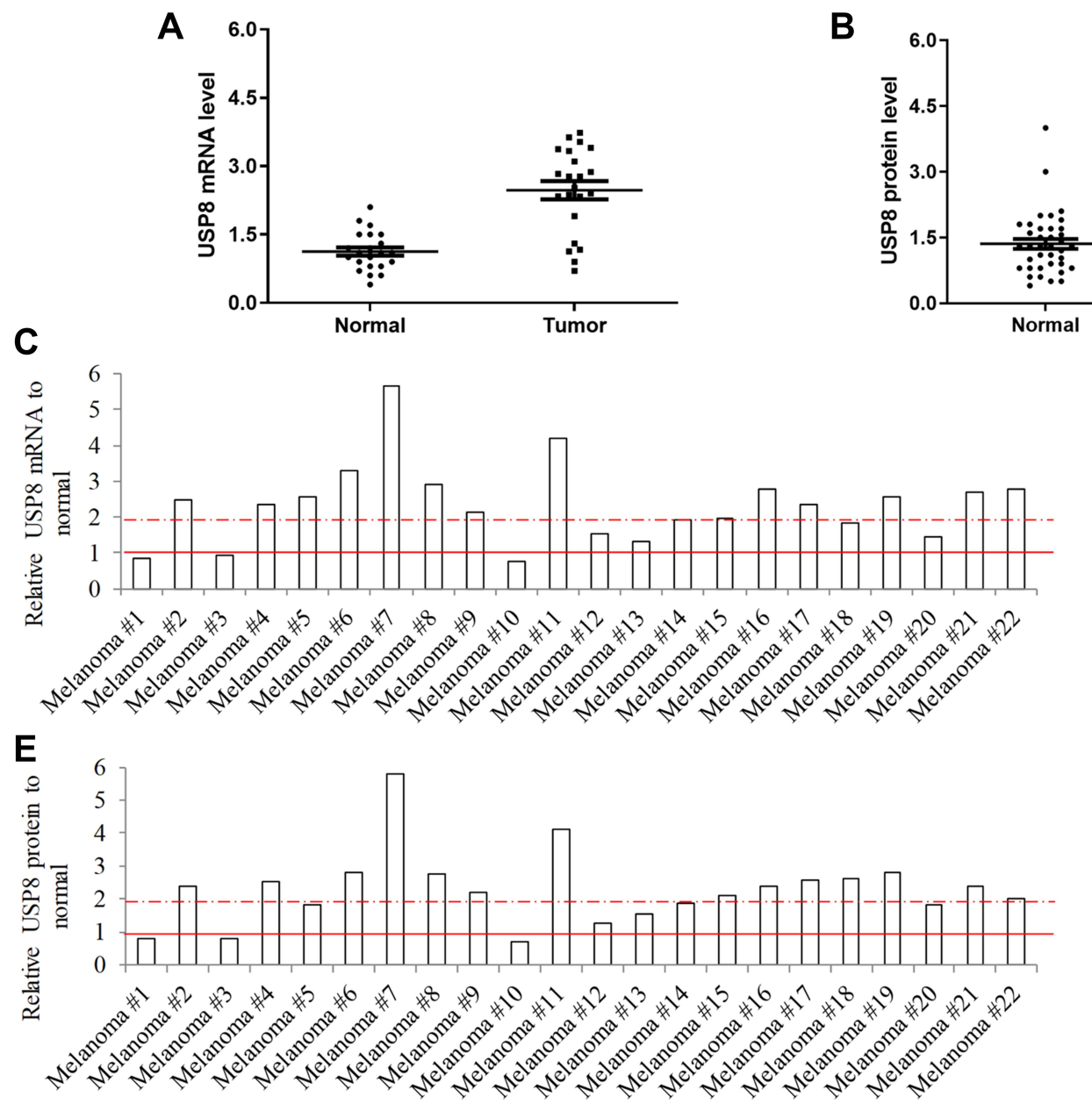

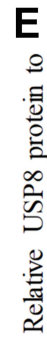
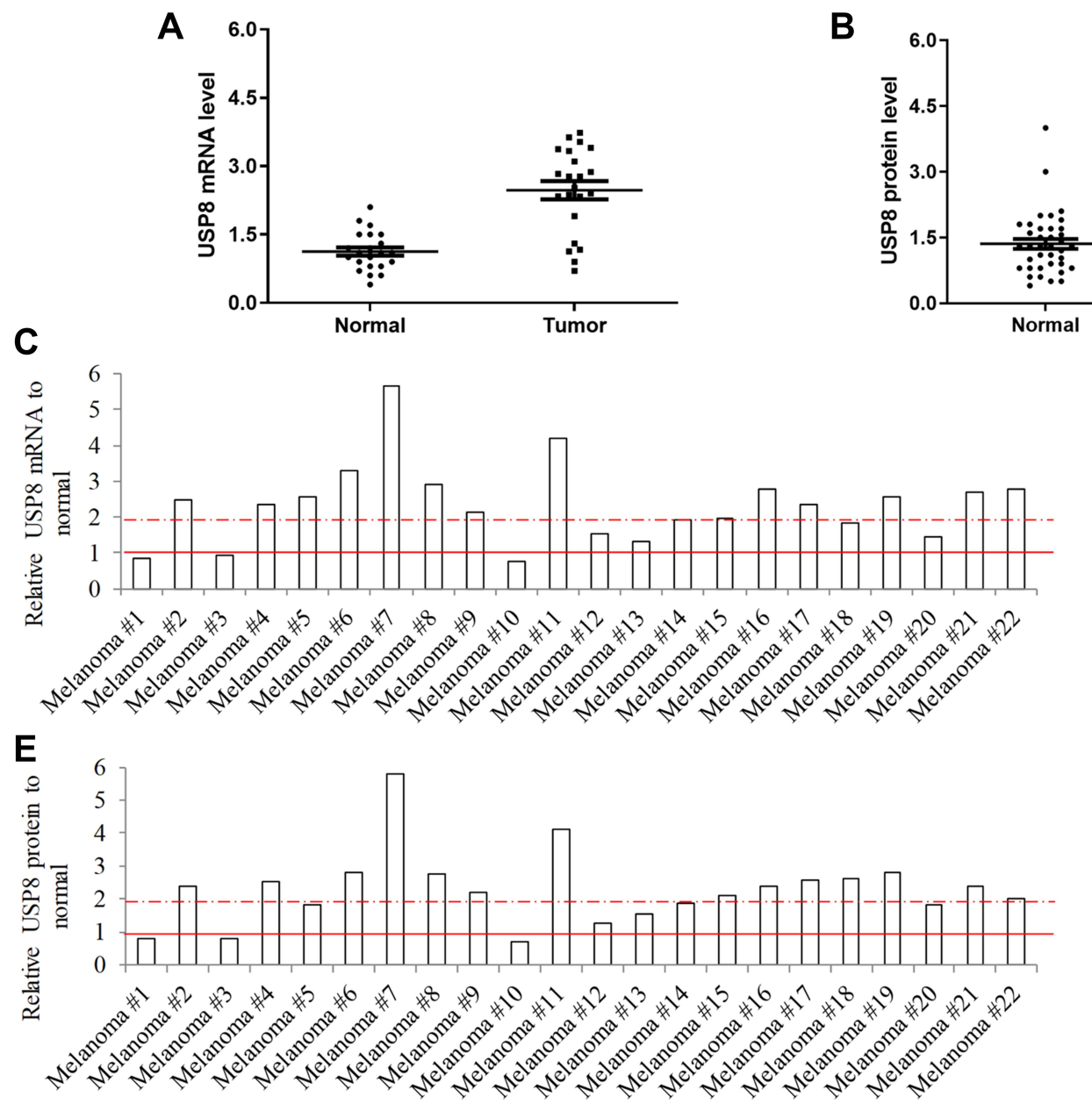

USP8 Overexpression Activates

\section{Melanoma Cell Activities and Promotes}

Resistance to Standard of Care Drugs

To investigate the biological function of USP8 in melanoma, UACC-62 cells with comparable USP8 level as normal melanocytes were chosen to be transfected with USP8overexpressing plasmid and confirmed with USP8 overexpression after transfection $(\mathrm{P}<0.05$; Figure $3 \mathrm{~A})$. We found that USP8 overexpression significantly increased UACC-62 cell growth and migration by 2 to 3 -fold ( $\mathrm{P}<0.05$; Figure $3 \mathrm{~B}-$ D). We next investigated the effects of USP8 overexpression in melanoma cell response to standard of care drugs: vemurafenib and dacarbazine. Dacarbazine is a chemotherapeutic agent ${ }^{16}$ and vemurafenib is a B-Raf inhibitor, ${ }^{17}$ and both are

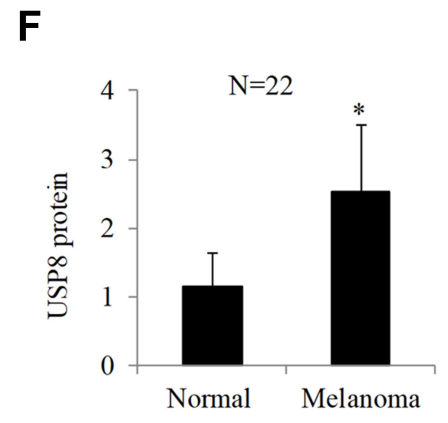

Figure 2 USP8 expression is significantly increased in melanoma patients. Scatter plot of mRNA (A) and protein (B) of USP8 in paired melanoma malignant and their adjacent normal tissues. Samples were obtained from 22 melanoma patients. The line shown in scatter plot indicates the average expression of USP8 in melanoma malignant and normal tissue. USP8 mRNA (C) and protein (E) ratio of tumor to normal in individual melanoma patients. Dot line and solid line indicate tumor/normal ratio at 2 and I, respectively. Average USP8 mRNA (D) and protein (F) levels in melanoma malignant and normal tissue. Equal amount of proteins from each sample were used in ELISA assay. Results were presented as fold change relative to normal. $* \mathrm{P}<0.05$, compared to normal. 
clinically used for the treatment of advanced stage of melanoma. We observed the significantly more proliferating and viable cells in USP8-ovexpressing cells than control cells after treatment of vemurafenib and dacarbazine $(\mathrm{P}<0.05$; Figure $3 \mathrm{E}$ and $\mathrm{F}$ ), demonstrating that USP8 overexpression results in resistance to standard of care drugs.

\section{USP8 Knockdown Suppresses Melanoma Cell Activities and Augments Efficacy of Standard of Care Drugs}

We further attempted to confirm the biological function of USP8 in melanoma using loss-of-function approach. UACC-257 and SK-MEL-28 with high USP8 expression level were chosen to perform to be transfected with USP8 siRNA. siRNA-control (siCtrl) was used as negative

A

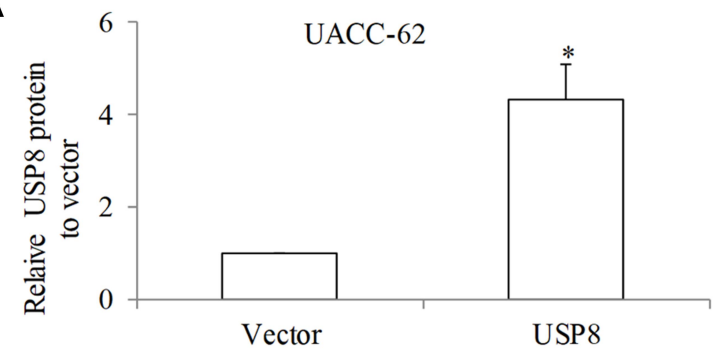

C

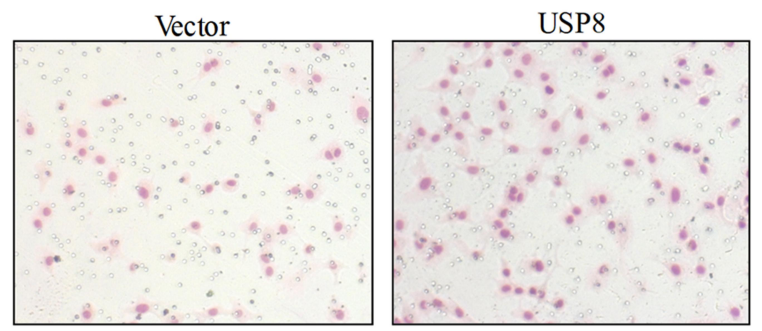

E

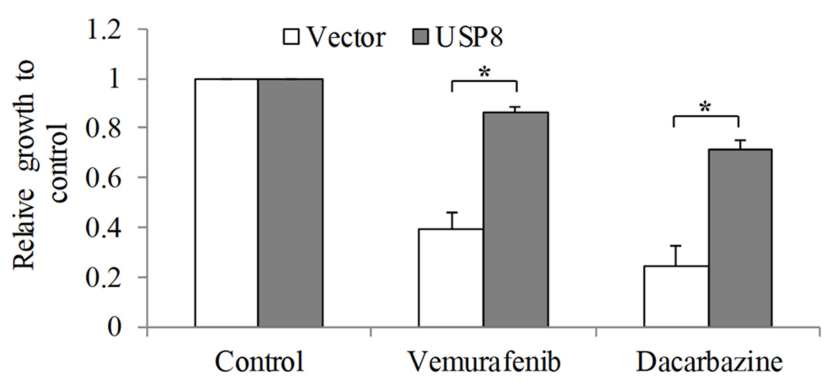

control. We used multiple siRNA and identified siUSP8\#1 and siUSP8\#2 as the most efficient that achieved almost complete USP8 depletion in melanoma cells (Supplementary Figure 2, Figure 4A and data not shown). As shown in Figure 4A and B, USP8 knockdown significantly decreased proliferation of both melanoma cell lines to $50 \%$ to $60 \%$. USP 8 inhibition also significantly increased apoptosis to $30 \%$ to $40 \%$, and inhibited migration to $50 \%$ to $60 \%(\mathrm{P}<0.05$; Figure $4 \mathrm{C}-\mathrm{F})$. Of note, vemurafenib and dacarbazine treatment resulted in significantly less proliferation and more apoptosis in USP8depleted cells than siCtrl cells $(\mathrm{P}<0.05$; Figure 4A-D). However, we did not observe the enhanced combinatory effect of USP8 knockdown and drug treatment on melanoma cell migration (Figure 4E and F).

B

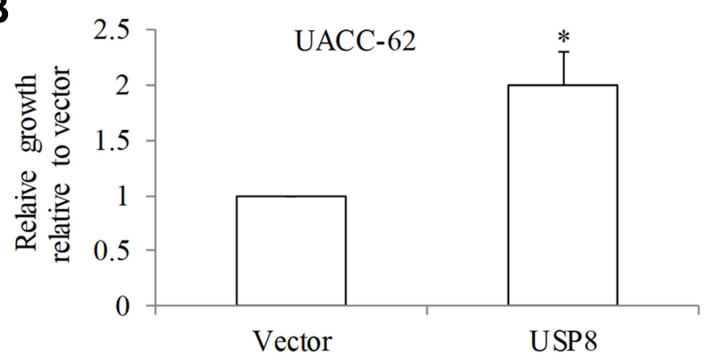

D

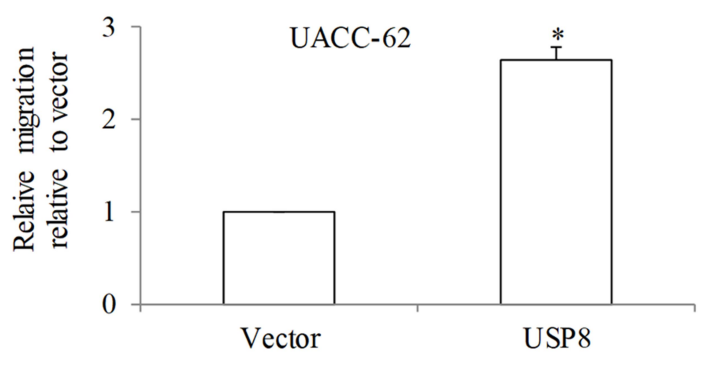

$\mathbf{F}$

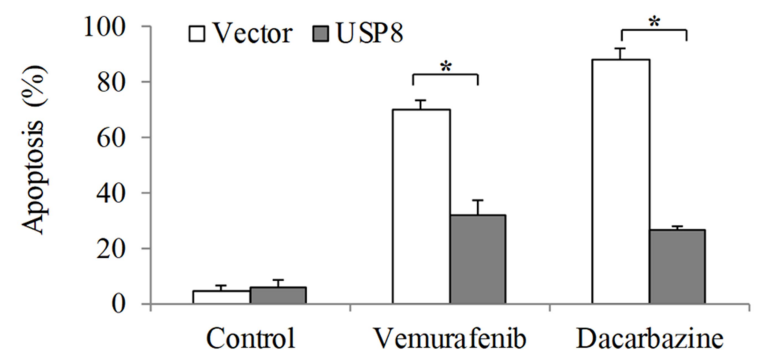

Figure 3 USP8 overexpression significantly promotes melanoma cell activities and alleviates efficacy of standard of care drugs. (A) USP8 protein level was significantly increased in USP8-overexpressing melanoma cells. (B) USP8 overexpression significantly augmented melanoma cell proliferation. Representative images (C) and quantification (D) of migration assay demonstrated enhanced migration in USP8-overexpressing melanoma cells. Migration quantification was performed by counting number of migrated cells from five selected fields. USP8 overexpression significantly reversed anti-proliferative (E) and pro-apoptotic (F) effects of vemurafenib (0.5 $\mu \mathrm{M})$ and dacarbazine $(10 \mu \mathrm{M})$ in UACC-62 cells. Results were presented as fold change relative to normal. $* \mathrm{P}<0.05$, compared to control. 

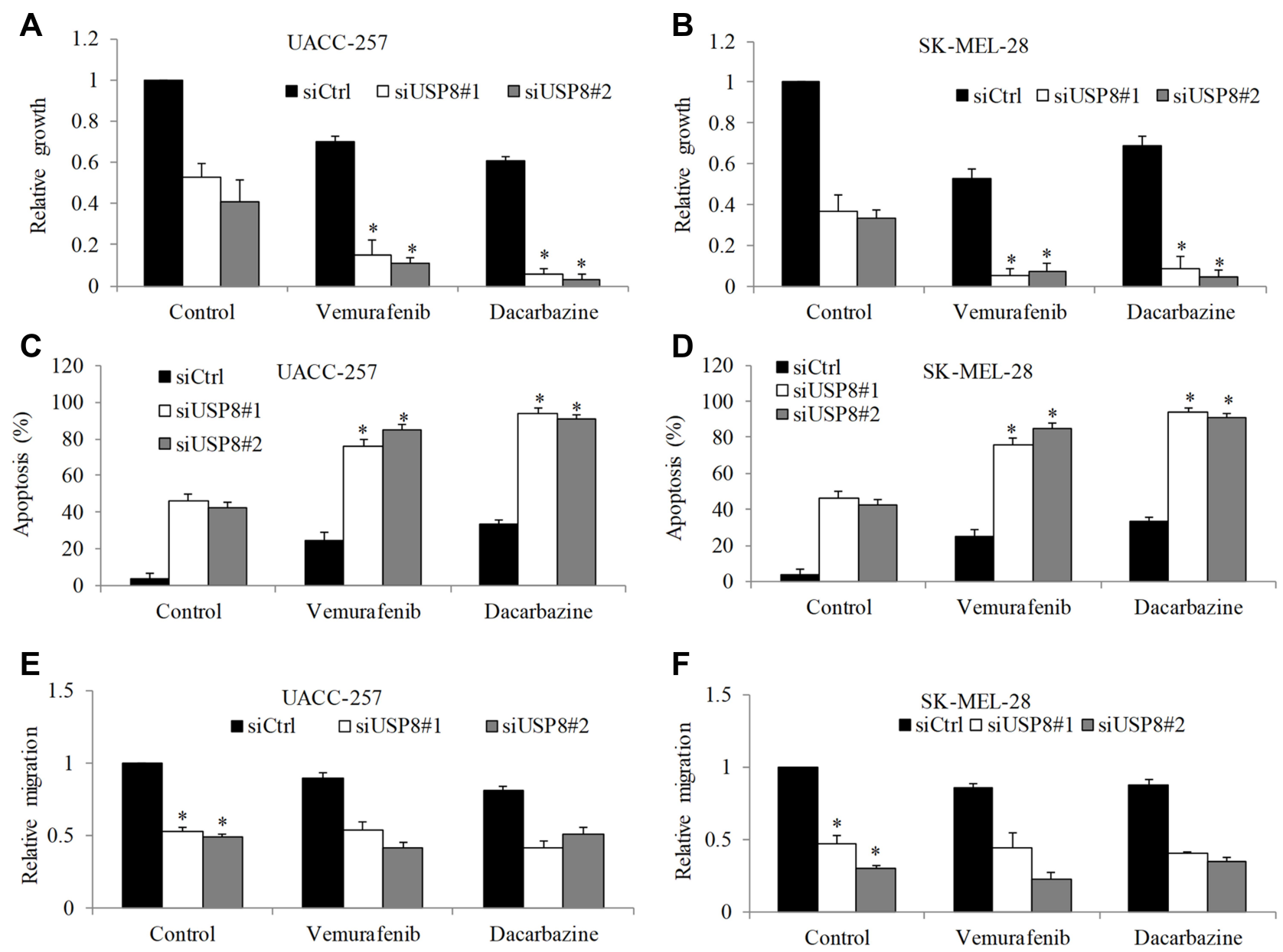

Figure 4 USP8 depletion significantly enhances the inhibitory effects of standard of care drug in melanoma cells. USP8 knockdown significantly decreased growth and increased apoptosis in UACC-257 (A and C) and SK-MEL-28 (B and D) cells, and enhanced anti-proliferative and pro-apoptotic effect of vemurafenib (0.I $\mu$ M) and dacarbazine (I $\mu \mathrm{M})$. USP8 knockdown significantly decreased migration in UACC-257 (E) and SK-MEL-28 (F) cells. Results were presented as fold change relative to control. $* \mathrm{P}<0.05$, compared to control.

\section{USP8 Depletion Decreases RTK Expression Levels and Suppresses RTK-Mediated Signaling Pathways in \\ Melanoma}

To investigate the underlying mechanisms of USP8 inhibition in melanoma, we examined the expression level of RTKs in USP8-depleted melanoma cells because 1) USP8 inhibition is known to reduce protein level of many RTKs in cancer cells; ${ }^{8,11}$ 2) RTKs and RTK-mediated signaling, particularly c-Met, Kit, EGFR and G-protein-coupled receptor (GPCR), play essential roles in melanoma., ${ }^{2,19}$ Western blotting analysis showed that USP8 inhibition remarkably decreased protein level of Met, EGFR, c-Kit (Figure 5A). In addition, USP8 inhibition decreased CYSLTR2 level without affecting ENDRA, indicating that USP8 decreases some but not all GPCR expression.
In line with the decreased level of RTKs, there was a decreased p-Stat3, p-Raf, p-p90RSK, p-FAK and p-Akt in USP8-depleted cells (Figure 5B), suggesting that USP8 inhibition suppresses RTK-mediated downstream Raf/ MEK, PI3K/Akt and FAK signaling pathways. Consistent with the increased apoptosis, USP8 depletion increased cleaved caspase 3 in melanoma cells (Figure 5C). Decreased anti-apoptotic molecules such as Mcl-1 and $\mathrm{Bcl}-\mathrm{xL}$, and increased pro-apoptotic molecules such as Bax, were detected in USP8-depleted cells. Bcl-2 level was not affected by USP8 inhibition.

\section{Discussion}

In this study, we are the first to provide a proof-of-concept that USP8 inhibition via genetic knockdown significantly enhances the inhibitory effects of dacarbazine and vemurafenib in melanoma cell growth and survival, suggesting 
A

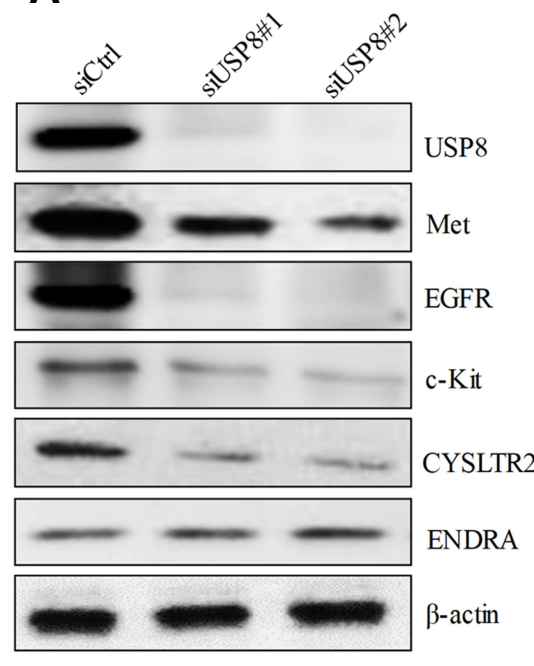

B

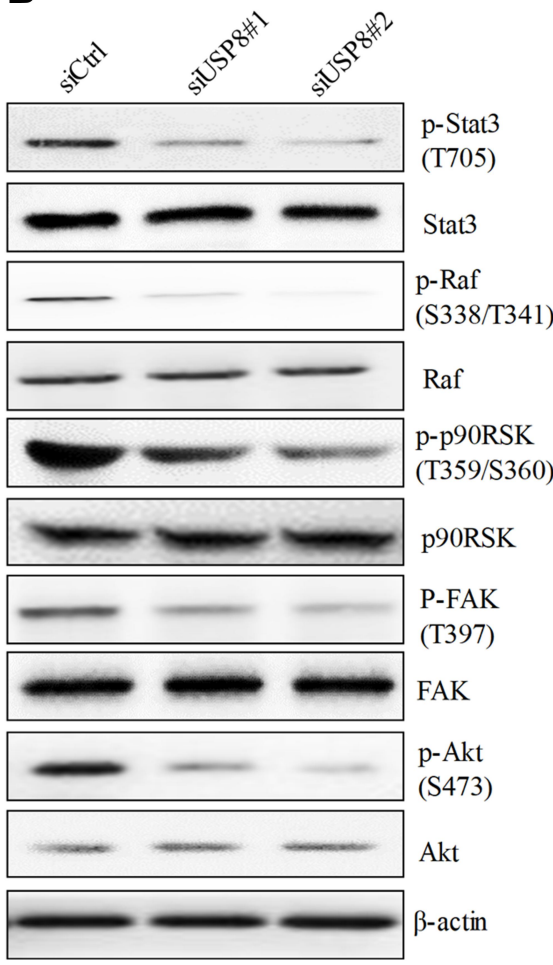

C

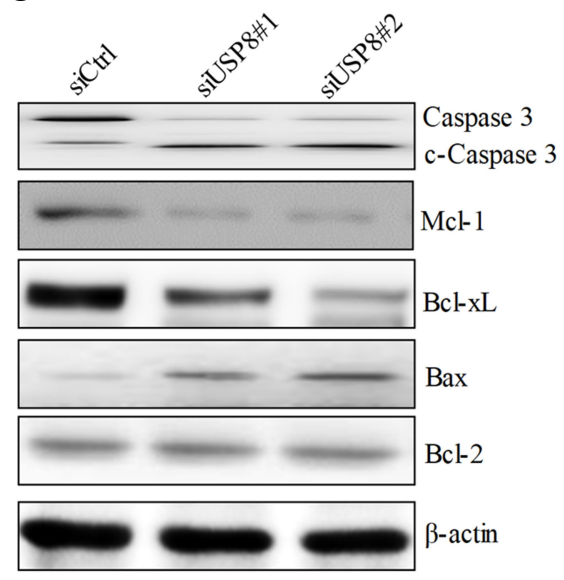

Figure 5 USP8 depletion decreases expression of RTK and inhibits RTK-mediated pathways in melanoma cells. (A) The inhibitory effect of USP8 depletion on the expression level of various RTKs in melanoma cells. (B) The inhibitory effect of USP8 depletion on the phosphorylation of molecules involved in RTK-mediated pathways in melanoma cells. (C) The effects of USP8 knockdown on the level of pro-apoptotic proteins (Bax and Bcl-2) and anti-apoptotic proteins (Mcl-I and Bcl-xL) in melanoma cells. Western blot was performed using cells at 48 hours post-transfection.

that USP8 is a promising therapeutic target to sensitize melanoma cells in response to dacarbazine and vemurafenib. USP8 belongs to a big USP family that their dysregulation are associated with development and drug resistance of several cancers. ${ }^{20}$ Except USP8, other USPs including USP4, USP10/13, USP7 and USP39, have been shown to play a role in melanoma growth, survival and metastasis. $^{21-24}$ Our work adds USP8 to the list of USPs that are critical to melanoma activities.

We show that USP8 mRNA and protein are increased in many melanoma cell lines and patients compared to their normal counterparts. This is consistent with the previous findings that USP10, 13, 4 and 39 are also significantly elevated in melanoma. ${ }^{21,23,24}$ In addition, USP4 expression in metastatic melanomas is higher than that in primary ones. ${ }^{24}$

According to the data from the Cancer Genome Atlas, it is noted that melanoma patients with high expression of USP8 display worse survival, suggesting the prognostic value of USP8 in melanoma. USP8 upregulation is associated with advanced tumor stage, high recurrence risk and poor outcome in patients with cervical cancer and lung adenocarcinoma. ${ }^{7,25}$ Based on our findings and published data, it will be significant to confirm the prognostic value of USP8 in melanoma.

The systematic functional analysis using multiple melanoma cell lines show that USP8 plays a critical role in melanoma cell growth, migration, survival and response to drug treatment. USP8 overexpression significantly promoted melanoma cell growth and migration. Consistently, USP8 depletion decreased cell proliferation and migration, and increased apoptosis. In addition, USP8 overexpression resulted in melanoma resistance to drug treatment whereas USP8 depletion augmented the efficacy of both drugs. These results correlate well with each other and support the investigation of therapeutic value of targeting USP8 using pharmacological inhibitor USP8i (9-ehtyloxyimino9H-indeno [1,2-b] pyrazine-2,3-dicarbonitrile ${ }^{11,26}$ on melanoma preclinical models. The oncogenic role of USP8 in other cancers, such as lung cancer, cervical cancer and Cholangiocarcinoma, have been reported. ${ }^{6-8}$ Our work together with the previous reports suggest that USP8 is a novel therapeutic target for cancer. 
Importantly, our work further demonstrates notable reduction of multiple RTKs level and the subsequent disruption of RTKs-mediated signaling pathways in USP8-depleted melanoma cells. Met, EGFR, c-Kit and GPCR are preferentially important TRKs in regulating abnormal signaling driving aberrant growth, survival, and drug resistance in melanoma and are rational therapeutic targets. ${ }^{13}$ We noted that USP8 inhibition had little impact on ENDRA level, suggesting that USP8 inhibition does not affect all but only some specific RTKs. The decreased p-Stat3, p-Akt, p-Raf, p-p90RSK and p-FAK observed in USP8-depleted melanoma cells are expected. Most of the signaling are dysregulated not only in melanoma and but also other cancers. Our findings on the suppressed signaling of multiple RTKs in USP8depleted melanoma cells demonstrate the ability of USP8 inhibition on the suppression of global essential oncogenic signaling pathways.

In conclusion, our results reveal that USP8 is significantly increased in melanoma and plays multiple positive roles in melanoma via regulating RTKs. Our work highlights the therapeutic value of targeting USP8 in melanoma and provides pre-clinical evidence of testing USP8 pharmacological inhibitor in melanoma.

\section{Ethics Approval and Consent to Participate}

This study was conducted in accordance with the Declaration of Helsinki. The study protocol was approved by the Ethic Committee of Xiangyang Central Hospital. A written informed consent was obtained from all participants.

\section{Consent for Publication}

Consent for publication was obtained from every individual whose data are included in this manuscript.

\section{Funding}

This work was supported by Hubei University of Medicine (No. 2017-43).

\section{Disclosure}

Baoxue Duan and Changying Wang are co-first authors in this study. All authors declare that this work is original and has not been submitted or published elsewhere. None of the authors have any financial disclosure or conflicts of interest.

\section{References}

1. Siegel RL, Miller KD, Jemal A. Cancer statistics, 2015. CA Cancer J Clin. 2015;65(1):5-29. doi:10.3322/caac.21254

2. Czarnecka AM, Bartnik E, Fiedorowicz M, Rutkowski P. Targeted therapy in melanoma and mechanisms of resistance. Int J Mol Sci. 2020;21(13):4576. doi:10.3390/ijms21134576

3. Antao AM, Tyagi A, Kim KS, Ramakrishna S. Advances in deubiquitinating enzyme inhibition and applications in cancer therapeutics. Cancers. 2020;12(6):1579. doi:10.3390/cancers12061579

4. Young MJ, Hsu KC, Lin TE, Chang WC, Hung JJ. The role of ubiquitin-specific peptidases in cancer progression. J Biomed Sci. 2019;26(1):42. doi:10.1186/s12929-019-0522-0

5. Love KR, Catic A, Schlieker C, Ploegh HL. Mechanisms, biology and inhibitors of deubiquitinating enzymes. Nat Chem Biol. 2007;3 (11):697-705. doi:10.1038/nchembio.2007.43

6. Jing X, Chen Y, Chen Y, et al. Down-regulation of USP8 inhibits cholangiocarcinoma cell proliferation and invasion. Cancer Manag Res. 2020;12:2185-2194. doi:10.2147/CMAR.S234586

7. Yan M, Zhao C, Wei N, Wu X, Cui J, Xing Y. High expression of ubiquitin-specific protease 8 (USP8) is associated with poor prognosis in patients with cervical squamous cell carcinoma. Med Sci Mon. 2018;24:4934-4943. doi:10.12659/MSM.909235

8. Byun S, Lee SY, Lee J, et al. USP8 is a novel target for overcoming gefitinib resistance in lung cancer. Clin Cancer Res. 2013;19 (14):3894-3904. doi:10.1158/1078-0432.CCR-12-3696

9. Baykara M, Yaman M, Buyukberber S, et al. Clinical and prognostic importance of XIAP and USP8 in advanced stages of non-small cell lung cancer. $J$ BUON. 2013;18(4):921-927.

10. Niendorf S, Oksche A, Kisser A, et al. Essential role of ubiquitin-specific protease 8 for receptor tyrosine kinase stability and endocytic trafficking in vivo. Mol Cell Biol. 2007;27 (13):5029-5039. doi:10.1128/MCB.01566-06

11. Jeong $\mathrm{CH}$. Inhibition of ubiquitin-specific peptidase 8 suppresses growth of gefitinib-resistant non-small cell lung cancer cells by inducing apoptosis. J Cancer Prev. 2015;20(1):57-63. doi:10.15430/ JCP.2015.20.1.57

12. Jian FF, Li YF, Chen YF, et al. Inhibition of ubiquitin-specific peptidase 8 suppresses adrenocorticotropic hormone production and tumorous corticotroph cell growth in AtT20 cells. Chin Med J. 2016;129(17):2102-2108. doi:10.4103/0366-6999.189047

13. Easty DJ, Gray SG, O'Byrne KJ, O’Donnell D, Bennett DC. Receptor tyrosine kinases and their activation in melanoma. Pigment Cell Melanoma Res. 2011;24(3):446-461. doi:10.1111/ j.1755-148X.2011.00836.x

14. Yang X, Peng X, Huang J. Inhibiting 6-phosphogluconate dehydrogenase selectively targets breast cancer through AMPK activation. Clin Transl Oncol. 2018;20(9):1145-1152. doi:10.1007/s12094-0181833-4

15. Vincent KM, Postovit LM. Investigating the utility of human melanoma cell lines as tumour models. Oncotarget. 2017;8 (6):10498-10509. doi:10.18632/oncotarget.14443

16. Serrone L, Zeuli M, Sega FM, Cognetti F. Dacarbazine-based chemotherapy for metastatic melanoma: thirty-year experience overview. J Exp Clin Cancer Res. 2000;19(1):21-34.

17. Bollag G, Tsai J, Zhang J, et al. Vemurafenib: the first drug approved for BRAF-mutant cancer. Nat Rev Drug Discov. 2012;11 (11):873-886. doi:10.1038/nrd3847

18. Pham DDM, Guhan S, Tsao H. KIT and melanoma: biological insights and clinical implications. Yonsei Med J. 2020;61 (7):562-571. doi:10.3349/ymj.2020.61.7.562

19. Gardner FP, Serie DJ, Salomao DR, et al. c-MET expression in primary and liver metastases in uveal melanoma. Melanoma Res. 2014;24(6):617-620. doi:10.1097/CMR.0000000000000118 
20. Tanguturi P, Kim KS, Ramakrishna S. The role of deubiquitinating enzymes in cancer drug resistance. Cancer Chemother Pharmacol. 2020;85(4):627-639. doi:10.1007/s00280-020-04046-8

21. Guo J, Zhang J, Liang L, et al. Potent USP10/13 antagonist spautin-1 suppresses melanoma growth via ROS-mediated DNA damage and exhibits synergy with cisplatin. J Cell Mol Med. 2020;24 (7):4324-4340. doi:10.1111/jcmm.15093

22. Vishnoi M, Boral D, Liu H, et al. Targeting USP7 identifies a metastasis-competent state within bone marrow-resident melanoma CTCs. Cancer Res. 2018;78(18):5349-5362. doi:10.1158/0008-5472. CAN-18-0644

23. Zhao Y, Zhang B, Lei Y, et al. Knockdown of USP39 induces cell cycle arrest and apoptosis in melanoma. Tumour Biol. 2016;37 (10):13167-13176. doi:10.1007/s13277-016-5212-x
24. Guo W, Ma J, Pei T, et al. Up-regulated deubiquitinase USP4 plays an oncogenic role in melanoma. J Cell Mol Med. 2018;22 (5):2944-2954. doi:10.1111/jcmm.13603

25. Kim Y, Shiba-Ishii A, Nakagawa T, et al. Ubiquitin-specific protease 8 is a novel prognostic marker in early-stage lung adenocarcinoma. Pathol Int. 2017;67(6):292-301. doi:10.1111/pin.12546

26. Colombo M, Vallese S, Peretto I, et al. Synthesis and biological evaluation of 9-oxo-9H-indeno[1,2-b]pyrazine-2,3-dicarbonitrile analogues as potential inhibitors of deubiquitinating enzymes. ChemMedChem. 2010;5(4):552-558. doi:10.1002/cmdc.200900409

\section{Publish your work in this journal}

Cancer Management and Research is an international, peer-reviewed open access journal focusing on cancer research and the optimal use of preventative and integrated treatment interventions to achieve improved outcomes, enhanced survival and quality of life for the cancer patient.
The manuscript management system is completely online and includes a very quick and fair peer-review system, which is all easy to use. Visit http://www.dovepress.com/testimonials.php to read real quotes from published authors. 